\title{
How do I tell a colleague they have made a mistake?
}

When a colleague makes a mistake it can be difficult to know how to approach them. Abi Rimmer seeks advice from three experts

\section{Abi Rimmer}

The BMJ

\section{"Get all the facts"}

Gabrielle Pendlebury, medicolegal consultant at Medical Protection, says, "To err is human. This can be uncomfortable to acknowledge within healthcare as errors, whether individual or systemic, can lead to patient harm. Mistakes, however, provide an opportunity to learn and stop similar errors from happening again. It's rare that a mistake on the part of a doctor is the sole cause of an adverse incident-there's usually some concurrent failure in the system or process. Many doctors, however, will avoid highlighting a colleague's error for fear of their reaction, the possible impact on their relationship, or for cultural reasons around loyalty and solidarity. The solution to this is to put patient safety and patient rights first, not your own professional worries. Professionalism should lead us to turn towards, rather than away from, a colleague who has made an error.

"The first obligation is to get the facts. Explore, do not ignore. Try and get the relevant information- the medical records may be lacking and events may have been misunderstood or misrepresented. Start with a colleague to colleague discussion of the problem in a non-judgmental way. This may be the first opportunity the clinician has to discuss the error in a supportive setting, and it could lead to considerable relief and comfort, as well as providing an opportunity to review the matter and reduce the risk of similar incidents occurring.

"From this first encounter, a joint decision can be made about next steps, whether this be disclosure to the institution or patients, with consideration of duty of candour; an apology; training; or review of systemic problems leading to changes in process.

"The use of a transparent collective approach is the only way to meet the needs of patients and families after harmful medical errors, and I suggest that a professional, caring perspective on these situations will aid that cultural shift."

\section{"Encourage them to learn from it"}

Nancy Redfern, consultant anaesthetist, says, "We all make mistakes; I've made many in my career. The dawning realisation that we've done something wrong is something we all dread.

"Unless it's an emergency requiring direct instruction-'Don't give that Augmentin injection; she's allergic to it!'-I start by trying to put myself in the other person's shoes. Conversations should always be private, held soon after the event and, where possible, on neutral ground. I explain what's happened and what my concerns are and ask the other person for their perspective.

"If it's clearly an error, such as forgetting to prescribe medication or to organise an event, then almost everyone is contrite. I often ask about the circumstances that led to the error-if they were distracted or too busy. The purpose of my questions is to help the person who has made the mistake identify the factors that led to it.

"Human factors literature makes clear that learning comes from having a better understanding of the whole situation, and that there are often systemic problems that allow or compound individual error.

"If we can identify these and work together to solve them it can help the individual feel more confident about owning up to mistakes in the future and possibly be less devastated by their error. And it may even improve the system.

"Matters are more complex when the person doesn't accept that they've made a mistake. Again, I try to give a clear explanation of what has happened, what I think should have happened, and why I am concerned. Summarising what the other person says is even more important here.

"If we can identify where we agree and where we differ, this helps separate the problem from the person. Looking at events from a different perspective, for example, 'I wonder what the patient would think,' can sometimes break an impasse. But sometimes we just have to agree to differ."

\section{"Be kind. Don't blame"}

Yasotha Browne, GP lead at the Doctors Association UK, says, "Keeping each other accountable is a tricky task and there are many variables. Assuming you are colleagues of equal footing, with a trusting relationship, and you noticed a minor error, it's important that you can approach them without blame.

"It's therefore essential to have a no blame culture in place before you need to have a conversation about error. This means cultivating a culture where everyone understands that errors should be reported to help identify stresses in the system and the working environment, not as a mechanism for apportioning blame. A good way to do this is by being forthcoming about your own mistakes. 
"We know that more mistakes happen when people are tired and stressed and, with already high rates of burnout among doctors, I would fully expect us to be seeing an increase in incident reports over the next five years.

"When approaching any discussion about an error, therefore, I would consider the context of that person's working environment, the access they have to education and training, and, if privy to the situation, what is going on at home.
Remember that marginalised groups, such as black and minority ethnic doctors, may be more fearful of being reprimanded.

"Finally, be kind. Don't be judgmental about the error as a way of promoting your own practice. I have observed this in many colleagues-stress and competitive environments can make us default to the worst of what we are.

"Morale among doctors is low and we need to do our best to build each other up. This includes a compassionate and safe attitude to discussing and managing error." 